\title{
Fixed-Time and Finite-Time Synchronization for a Class of Output-Coupling Complex Networks via Continuous Control
}

\author{
Zhiwei Li \\ College of Science, University of Shanghai for Science and Technology, Shanghai, China \\ Email: Z_W_Li_USST1@163.com
}

How to cite this paper: Li, Z.W. (2019) Fixed-Time and Finite-Time Synchronization for a Class of Output-Coupling Complex Networks via Continuous Control. Int. J. Communications, Network and System Sciences, 12, 151-169.

https://doi.org/10.4236/ijcns.2019.1210011

Received: September 21, 2019

Accepted: October 15, 2019

Published: October 18, 2019

Copyright $\odot 2019$ by author(s) and Scientific Research Publishing Inc. This work is licensed under the Creative Commons Attribution International License (CC BY 4.0). http://creativecommons.org/licenses/by/4.0/

\begin{abstract}
This paper mainly investigates the finite-time and fixed-time synchronization problem for a class of general output-coupling complex networks with output feedback nodes. The fixed-time and finite-time synchronization protocols are presented based on continuous controller strategies which can efficaciously eliminate chattering phenomenon existing in some previous results. Several sufficient conditions ensuring fixed-time and finite-time synchronization are derived by employing Lyapunov stability theory, linear matrix inequality (LMI) and adaptive technique. Furthermore, aimed at the model of this article, we study the problem of adaptive coupling strength in fixed-time synchronization which is rarely involved in previous results. Finally, several numerical examples are given to illustrate the effectiveness of our results.
\end{abstract}

\section{Keywords}

Output-Coupling Complex Networks, Fixed-Time Synchronization, Finite-Time Synchronization, Continuous Controller

\section{Introduction}

Over the past few decades, researches about complex dynamical networks have become more and more hot in many fields for its large-scale practicability such as information processing, World Wide Web, biological systems, neural networks and so on [1] [2] [3] [4]. In particular, the synchronization phenomenon considered as a significant collective behavior has been attracted rapidly extensive attention and many excellent works have been reported in [5]-[11] and the references therein.

Regarded as a fundamental problem of cooperative control, synchronization 
requires all subsystems to converge to a target state or a common value. It is worthwhile to note that most of the existing works about synchronization problems of complex dynamical networks were asymptotic synchronization [5]-[11], or exponential synchronization [12] [13], which meant that the synchronization could only be actualized within infinite time. In [5], the synchronization problem of general complex networks under pinning control was considered. Synchronization of an array of linearly coupled memristor-based recurrent neural networks with impulses and time-varying delays was investigated in [6]. In [9], the cluster synchronization problems of linearly coupled complex networks were studied by employing pinning control strategy. The authors of [12] studied the exponential synchronization problems of complex-valued complex networks with time-varying delays and stochastic perturbations via time-delayed impulsive control and coupled stochastic memristor-based neural networks with time-varying probabilistic delay coupling and impulsive delay [13]. However, in practice, we always expect that the synchronization could be achieved as quickly as possible indicating that the system can realize synchronization in a particular time period. This can be acquired by utilizing finite-time synchronization strategy proposed in [14] which has been demonstrated with many remarkable advantages including better robustness, higher precision and faster convergence rate, etc. For its prominent effect in improving convergence rate, many finite-time synchronization results were presented. [14] investigated the problem of finite-time synchronization for complex networks with nonidentical discontinuous nodes. In [15] results concerning the overlapping cluster finite-time synchronization of coupled complex networks via adaptive control were presented and its results can be applied to common cluster synchronization without overlap. The authors of [16] and [17] considered the finite-time synchronization of complex dynamical networks by periodically intermittent control and aperiodically intermittent control respectively. In [18], the finite-time cluster synchronization problem for complex networks were discussed by employing pinning control. Nevertheless, in the above results, the settle-time of finite-time synchronization heavily depends on the initial conditions of all the subsystems yet in some case, the information or knowledge of initial states is unknown or not available in advance. Furthermore, the settling time will be sufficiently large if the initial conditions are very large. These restrictions may limit its wide application in reality to some extent.

Whereupon, to work these constraints out, the fixed-time synchronization was proposed in which the settling time of fixed-time synchronization is bounded by a constant for arbitrary initial conditions and a lot of corresponding researches were done in [19] [20]. In [19], fixed-time cluster synchronization problem of general complex dynamical networks was considered with or without pinning control. In [19], the fixed-time synchronization theory was utilized to investigate complex networks with nonidentical nodes and stochastic noise perturbations and a new method which can be used to estimate the settling time was presented. 
In [20], based on event-triggered control strategy which can significantly reduce energy consumption and the frequency of the controller updates, the fixed-time synchronization was achieved in term of multi-agent systems with nonlinear uncertainties.

From the viewpoint of coupling among nodes, complex networks mainly be divided into two representative types, that is, output-coupling complex networks and state-coupling complex networks. In [21], global asymptotic adaptive synchronization problem for a class of output-coupling complex networks was studied by employing adaptive dynamical output feedback controllers. In recent years, most of researches focus on state-coupling complex networks while output-coupling complex networks attracts relatively less attention let alone research on fixed-time and finite-time synchronization problems.

In [22] [23], controllers, unexceptionally, include sign function which plays a very important and indispensable role in fixed-time and finite-time synchronization problems. However, some defects were brought such as chattering phenomenon [22] [23] mainly caused by the discontinuity of sign function which will do harm to the property of apparatus. Thus, in [10], to overcome these drawbacks and weaken chattering effect, a saturation function was utilized yet actually this effect cannot be intrinsically avoided. In [15], an adaptive controller which did not contain sign function was designed, and the chattering phenomenon was easily and successfully erased. However, the controller mentioned in [15] was still discontinuous, namely, the chattering effect still existed in controller. In [24], a controller excluding sign function was designed, however, the controller in [24] is still discontinuous.

As everyone knows, coupling strength among the nodes of complex networks plays a very significant role in the problem for the synchronization problem of complex networks. In general, by strengthening the effect of coupling to realize the synchronization for complex networks is a basic and prime idea. However, under this condition, the coupling strength is always expected to be as large as possible. Nonetheless, in practice, it is impractical that the coupling strength can be arbitrarily enhanced large. On the one hand, the complex networks with too large coupling strength cannot describe the real system. On the other hand, strengthening the effect of coupling strength, to some degree, may increase the cost in engineering field. Therefore, it is necessary and desirable to find a suitable and applicable coupling strength. A natural and effective way to realize this goal is to utilize adaptive technique [9]. In [25], the author studied the problem of synchronization of coupled connected neural networks with delays and the results showed that the theoretical value is usually larger than the value needed in practice. In [26], pinning control for complex networks by a single controller was investigated, but the coupling strength was required to be very large, which was very rigorous and not practical. In [27] [28] [29], the problem of adaptive coupling strength is not taken into consideration. To our best knowledge, the adaptive coupling strength problem for fixed-time synchronization of complex 
networks with output nodes is less investigated. In this paper, we will explore this problem deeply.

Motivated by the above discussion, the main novelties are enumerated as follows: 1) the fixed-time and finite-time synchronization problem for a more general complex dynamical networks with output feedback nodes are studied; 2) to avoid chattering phenomenon existing in previous works, a continuous controller is designed to realize synchronization; 3 ) the synchronization criterions in this paper are suitable for directed and undirected complex networks; 4) the adaptive coupling strength problem for fixed-time synchronization of complex networks with output nodes is studied first time.

The rest of this paper is organized as follows. In Section 2, the model of output-coupling complex networks, some lemmas, assumptions and definition are presented. The sufficient criterions ensuring fixed-time synchronization and finite-time synchronization of complex networks are derived in Section 3 and Section 4 respectively. In Section 5, the adaptive coupling strength problem for fixed-time synchronization of complex networks is investigated. In section 6, some numerical simulation examples are given.

\section{Problem Formulation and Preliminaries}

Notations: $R^{n}$ and $R^{n \times n}$ denote the set of $n$-dimension vector and $n \times n$ real matrix respectively. The superscript " $T$ " denotes the transpose of vectors and matrices. For the matrix $A \in R^{n \times n}, A^{s}=\frac{A+A^{\mathrm{T}}}{2}, \lambda_{\min }(A)$ and $\lambda_{\max }(A)$ denote the minimum eigenvalue and maximum eigenvalue of matrix $A$, respectively. Let $\mathbb{N}=\{1,2, \cdots, N\}$. $\operatorname{diag}\left[\alpha_{i}\right]_{n} \in R^{n \times n}$ is the diagonal matrix with diagonal entries $\alpha_{i}(i=1,2, \cdots, n) . I_{n} \in R^{n \times n}$ is the identity matrix. For the vector $x=\left[x_{1}, \cdots, x_{n}\right]^{\mathrm{T}}, x^{p}=\left[x_{1}^{p}, \cdots, x_{n}^{p}\right]^{\mathrm{T}}$. The Euclidean norm in $R^{n}$ is denoted as $\|\cdot\|$, i.e. $\|u\|=\sqrt{u^{\mathrm{T}} u}$ for $u \in R^{n}$.

In this paper, we consider the following output-coupling complex networks with output nodes:

$$
\left\{\begin{array}{l}
\dot{x}_{i}(t)=A x_{i}(t)+f\left(y_{i}(t)\right)+c \sum_{j=1}^{N} g_{i j} \Gamma y_{i}(t)+u_{i}(t), \\
y_{i}(t)=C x_{i}(t), \quad i \in \mathbb{N}
\end{array}\right.
$$

where $x_{i}(t) \in R^{n}$ is the system state variables of the $i$ th dynamical node, $y_{i}(t) \in R^{m}$ is the output variables of the $i$ th dynamical node, $f: R^{n} \rightarrow R^{n}$ is a continuous function governing the dynamics of $i$ th isolated nodes, $c>0$ is a coupling strength, $G=\left[g_{i j}\right]_{N \times N}$ is the weight configuration matrix of the complex networks, $\Gamma \in R^{n \times m}$ is the inner coupling matrix and $C \in R^{m \times n}$ is the output matrix, $u_{i}(t) \in R^{n}$ is the controller designed for the system (1). For $i \neq j, \quad g_{i j}>0$ if and only if there is a connection from node $i$ to the node $j$, and the diagonal elements are defined as 


$$
g_{i i}=-\sum_{j=1, j \neq i}^{N} g_{i j} .
$$

The initial value of the complex network (1) is $x_{i}(0)=x_{i 0}, i \in \mathbb{N}$.

Remark 1: Here, we assume that the weight configuration matrix $G$ is not symmetric and irreducible. Therefore, the system (1) can be used to describe the directed and the undirected weighted complex networks.

Remark 2: When the output matrix C is the identity matrix $I_{N}$, then (1) degrade into

$$
\dot{x}_{i}(t)=A x_{i}(t)+f\left(x_{i}(t)\right)+c \sum_{j=1}^{N} g_{i j} \Gamma x_{i}(t)+u_{i}(t)
$$

which has been deeply studied by [18] [19]. Therefore, system (1) is more general than system (2).

The goal of this paper is to design suitable controllers $u_{i}(t)$ such that the states of complex network (1) synchronize into the state of following target system within a finite time and fixed time,

$$
\left\{\begin{array}{l}
\dot{x}^{*}(t)=A x^{*}(t)+f\left(y^{*}(t)\right) \\
y^{*}(t)=C x^{*}(t)
\end{array}\right.
$$

Subtracting (3) from (1), the following error dynamical systems are obtained:

$$
\left\{\begin{array}{l}
\dot{e}_{i}(t)=A e_{i}(t)+F\left(\psi_{i}(t)\right)+c \sum_{j=1}^{N} g_{i j} \Gamma \psi_{j}(t)+u_{i}(t), \\
\psi_{i}(t)=y_{i}(t)-y^{*}(t), \quad i \in \mathbb{N}
\end{array}\right.
$$

where

$$
e_{i}(t)=x_{i}(t)-x^{*}(t), F\left(\psi_{i}(t)\right)=f\left(y_{i}(t)\right)-f\left(y^{*}(t)\right),
$$

$e_{i}(t)$ and $\psi_{i}(t)$ are the state error and output error of the $i$ th node, respectively. Obviously, we have

$$
\begin{gathered}
\psi_{i}(t)=C x_{i}(t)-C x^{*}(t)=C e_{i}(t) . \\
\text { Let } e(t)=\left[e_{1}^{\mathrm{T}}(t), e_{2}^{\mathrm{T}}(t), \cdots, e_{N}^{\mathrm{T}}(t)\right]^{\mathrm{T}} .
\end{gathered}
$$

Definition 1: The network (1) is said to be synchronized onto (3) within a finite time, if there exists a settling time $T>0$ which is dependent on the initial value, such that

$$
\|e(t)\| \equiv 0 \text { for all } t \geq T,
$$

where $e(t)$ is the solution of error dynamical system (4).

Definition 2: The network (1) is said to be synchronized onto (2) within a fixed time, if there exists a settling time $T>0$ which is independent on the initial value, such that

$$
\|e(t)\| \equiv 0 \text { for all } t \geq T,
$$

where $e(t)$ is the solution of error dynamical system (8). 
Lemma 1: [21] If $A \in R^{n \times n}$ is a real symmetric matrix, then

$$
\lambda_{\text {min }}(A) x^{\mathrm{T}} x \leq x^{\mathrm{T}} A x \leq \lambda_{\max }(A) x^{\mathrm{T}} x \text {, for any } x \in R^{n} .
$$

Lemma 2: [14] Suppose that a continuous, positive-definite function $V(t)$ satisfies the following inequality:

$$
\dot{V}(t) \leq-\alpha V^{p}(t), \forall t \geq 0
$$

where $\alpha>0,0<p<1$. Then, for any given $t_{0}$,

$$
V(t) \equiv 0, \forall t \geq T\left(x_{0}\right),
$$

where

$$
T\left(x_{0}\right)=\frac{V^{1-p}(0)}{\alpha(1-p)} .
$$

Lemma 3: [29] Suppose that a continuous, positive-definite function $V(t)$ satisfies the following inequality:

$$
\dot{V}(t) \leq-\beta V^{p}(t)-\gamma V^{q}(t)
$$

where $\beta>0, \gamma>0,1>p>0, q>1$. Then, $V(t) \equiv 0, \forall t \geq T$ with

$$
T=\frac{1}{\beta(1-p)}+\frac{1}{\gamma(q-1)} \text {. }
$$

Lemma 4: If $\xi_{1}, \xi_{2}, \cdots, \xi_{n} \geq 0,0<\imath \leq 1, \kappa>1$, then

$$
\sum_{i=1}^{n} \xi_{i}^{l} \geq\left(\sum_{i=1}^{n} \xi_{i}\right)^{l} \text { and } \sum_{i=1}^{n} \xi_{i}^{\kappa} \geq n^{1-\kappa}\left(\sum_{i=1}^{n} \xi_{i}\right)^{\kappa} .
$$

Assumption 1: [26] The function $f(*)$ is said to satisfy the lipschitz condition if there exists a positive constant $\delta$ such that

$$
\|f(x)-f(y)\| \leq \delta\|x-y\| .
$$

\section{Finite-Time Synchronization}

In this section, we will design suitable controllers $u_{i}(t)$ such that output-coupling complex networks (1) synchronize into (3) with finite time. In order to realize this control goal, the controllers are designed as:

$$
u_{i}(t)=-\epsilon_{i} e_{i}(t)-\beta e_{i}^{\frac{\theta}{\phi}}(t), i \in \mathbb{N},
$$

where $\epsilon_{i}>0 \quad(i \in \mathbb{N}), \theta$ and $\phi$ are positive odd integers satisfying $\theta<\phi$ to be determined, and $\beta>0$ is a tunable constant.

Remark 3: In the previous works, the following controllers were often employed in the process of realizing finite-time synchronization:

$$
u_{i}(t)=-\eta_{1} e_{i}(t)-\eta_{2} \operatorname{sign}\left(e_{i}(t)\right)-\eta_{3} \operatorname{sign}\left(e_{i}(t)\right)\left|e_{i}(t)\right|^{\frac{\theta}{\phi}}
$$

and

$$
u_{i}(t)=-\eta_{1} \operatorname{sign}\left[h\left(x_{i}(t)\right)-h\left(x_{0}(t)\right)\right]-\eta_{2} \operatorname{sign}\left[h\left(x_{i}(t)\right)-h\left(x_{0}(t)\right)\right]^{\frac{\theta}{\phi}},
$$


where $\eta_{1}, \eta_{2}, \eta_{3}$ are the positive control strength and $h$ is vector valued function. Apparently, above controllers were discontinuous which may introduce the chattering problem and other superfluous behavior to the states of system and control messages frequently.

Remark 4: The controllers (7) which exclude sign function are continuous. Therefore, the chattering phenomenon can be avoided under the controller (7).

Theorem 1: Suppose that Assumption 1 holds. If there exist positive constants $\epsilon_{i} \in \mathbb{N}$ such that

$$
\left(\lambda_{\max }\left(A^{s}\right)+\delta \chi\right) I_{N}+G^{*}-\Pi \leq 0,
$$

where $\chi=\sqrt{\lambda_{\max }\left(C^{\mathrm{T}} C\right)}, \Pi=\operatorname{diag}\left[\epsilon_{i}\right]_{N}, G^{*}=\left[g_{i j}^{*}\right]_{N \times N}$, with $g_{i i}^{*}=c \varpi g_{i i}$ and $g_{i j}^{*}=\operatorname{c\varrho g}_{i j}(i \neq j)$, where $\varpi=\sqrt{\lambda_{\max }(\Gamma C)^{s}}, \varrho=\|\Gamma C\|$. Then, the controlled network (1) is synchronized onto the target state (3) under the controller (7) in a finite time:

$$
T_{0}=\frac{2 \phi V^{\frac{\phi-\theta}{2 \phi}}(0)}{\beta 2^{\frac{\phi+\theta}{2 \phi}}(\phi-\theta)},
$$

where $V(0)=\frac{1}{2} \sum_{i=1}^{N} e_{i}^{\mathrm{T}}(0) e_{i}(0)$.

Proof: Define a Lyapunov function:

$$
V=\frac{1}{2} \sum_{i=1}^{N} e_{i}^{\mathrm{T}}(t) e_{i}(t)
$$

Calculating the derivative of $V(t)$ along the trajectory of system (4), it follows that

$$
\begin{aligned}
\dot{V}(t)= & \sum_{i=1}^{N} e_{i}^{\mathrm{T}}(t) A^{s} e_{i}(t)+\sum_{i=1}^{N} e_{i}^{\mathrm{T}}(t) F\left(\psi_{i}(t)\right)+c \sum_{i=1}^{N} \sum_{j=1}^{N} g_{i j} e_{i}^{\mathrm{T}}(t) \Gamma \psi_{j}(t) \\
& -\sum_{i=1}^{N} \epsilon_{i} e_{i}^{\mathrm{T}}(t) e_{i}(t)-\beta \sum_{i=1}^{N} e_{i}^{\mathrm{T}}(t) e_{i}^{\frac{\theta}{\phi}}(t) .
\end{aligned}
$$

Based on Lemma 1 Equation (9) and Assumption 1, we have

$$
\begin{aligned}
\dot{V}(t) \leq & \lambda_{\max }\left(A^{s}\right) \sum_{i=1}^{N}\left\|e_{i}(t)\right\|^{2}+\delta \chi \sum_{i=1}^{N}\left\|e_{i}(t)\right\|^{2}+c \varpi \sum_{i=1}^{N} g_{i i}\left\|e_{i}(t)\right\|^{2} \\
& +c \varrho \sum_{i=1}^{N} \sum_{j=1, j \neq i}^{N} g_{i j}\left\|e_{i}(t)\right\| \cdot\left\|e_{j}(t)\right\|-\sum_{i=1}^{N} \epsilon_{i}\left\|e_{i}(t)\right\|^{2}-\beta 2^{\frac{\phi+\theta}{2 \phi}} V^{\frac{\phi+\theta}{2 \phi}}(t) .
\end{aligned}
$$

Denote $E^{\mathrm{T}}=\left[\left\|e_{1}(t)\right\|,\left\|e_{2}(t)\right\|, \cdots,\left\|e_{N}(t)\right\|\right]$, then

$$
\dot{V}(t) \leq E^{\mathrm{T}}\left\{\left(\lambda_{\max }\left(A^{s}\right)+\delta \chi\right) I_{N}+G^{*}-\Pi\right\} E-\beta 2^{\frac{\phi+\theta}{2 \phi}} V^{\frac{\phi+\theta}{2 \phi}}(t)
$$

By (10), it follows that

$$
\dot{V}(t) \leq-\beta 2^{\frac{\phi+\theta}{2 \phi}} V^{\frac{\phi+\theta}{2 \phi}}(t)
$$

By utilizing Lemma 2, we achieve $\lim _{t \rightarrow T_{0}}\left\|e_{i}(t)\right\|=0, i=1,2, \cdots, N$, and the finite time is estimated by 


$$
T_{0}=\frac{2 \phi V^{\frac{\phi-\theta}{2 \phi}}(0)}{\beta 2^{\frac{\phi+\theta}{2 \phi}}(\phi-\theta)} .
$$

The proof is completed.

Especially, if the output matrix $C$ is an identity matrix with appropriate dimensions, then we have following result.

Corollary 1: Suppose that Assumption 1 holds and $C=I_{N}$. If there exist positive constants $\epsilon_{i} \in \mathbb{N}$ such that

$$
\left(\lambda_{\max }\left(A^{s}\right)+\delta\right) I_{N}+\hat{G}^{*}-\Pi \leq 0 .
$$

where $\Pi=\operatorname{diag}\left[\varepsilon_{i}\right]_{N}$ and $\hat{G}^{*}=\left(\hat{g}_{i j}^{*}\right)$ with $\hat{g}_{i i}^{*}=c \hat{\omega} g_{i i}$ and $\hat{g}_{i j}^{*}=c \hat{\varrho} g_{i j}(i \neq j)$, where $\hat{\omega}=\sqrt{\lambda_{\max }\left(\Gamma^{s}\right)}$ and $\hat{\varrho}=\|\Gamma\|$. Then complex network (1) can achieve finite-time synchronization under the controller (7).

Corollary 2: Suppose that Assumption 1 holds, the inner coupling matrix $\Gamma=I_{N}$, output matrix $C=I_{N}$ and coupling strength $c=1$. If there exists a positive constant $\varepsilon$ such that

$$
\left(\lambda_{\max }\left(A^{s}\right)+\delta-\epsilon\right) I_{N}+G \leq 0,
$$

where $G$ is the weight configuration matrix of the complex networks. Then complex network (1) can achieve finite-time synchronization under the controller (7).

\section{Fixed-Time Synchronization}

In this section, we will design suitable controllers $u_{i}(t)$ such that output-coupling complex networks (1) synchronize into the target state (3) within a fixed settling time. In order to realize this goal, we suppose

$$
u_{i}(t)=-\xi_{i} e_{i}(t)-\beta e_{i}^{\frac{\theta}{\phi}}(t)-\gamma e_{i}^{\frac{k}{l}}(t),
$$

where $\theta, \phi, k, l$ are all positive odd integers satisfying $\theta>\phi$ and $k<l$.

Remark 5: Obviously, the controllers (11) are continuous and the sign function is excluded as well. As is well known, the role played by sign function in controller is pretty significant, however, it always introduce chattering phenomenon to the system and controller which may damage the devices. In literature [15] [18] [22] [27], the controller is composed of sign function and state error in studying the synchronization problems. In this paper, without utilizing the sign function in the controller, the chattering phenomenon is erased successfully.

Remark 6: Referring to [27], a more precise estimated value of settling time can be obtained by utilizing the controller (11).

Remark 7: Some discontinuous controllers designed by signal function and error function like

$$
u_{i}(t)=-\xi_{i} e_{i}(t)-\eta_{i} \operatorname{sign}\left(e_{i}(t)\right)-\beta \operatorname{sign}\left(e_{i}(t)\right)\left|e_{i}(t)\right|^{p}-\gamma \operatorname{sign}\left(e_{i}(t)\right)\left|e_{i}(t)\right|^{q},
$$

where $p$ and $q$ are positive constants satisfying $0<p<1$ and $q>1$. 


$$
u_{i}(t)=-\xi_{i} e_{i}(t)-\eta_{i} \operatorname{sign}\left(e_{i}(t)\right)-\beta \operatorname{sign}\left(e_{i}(t)\right)\left|e_{i}(t)\right|^{\frac{m}{h}}-\gamma \operatorname{sign}\left(e_{i}(t)\right)\left|e_{i}(t)\right|^{\frac{k}{h}},
$$

where $m, h, k, l$ are all positive odd integers satisfying $m>h$ and $k<l$, can be employed in the issue of fixed-time synchronization of complex networks. However, the chattering problem and other superfluous behavior may be brought to the states of system and control messages frequently.

Theorem 2: Suppose that Assumption1 holds. If there exists positive constants $\xi_{i}, i=1,2, \cdots, N$, such that

$$
\left(\lambda_{\max }\left(A^{s}\right)+\delta \chi\right) I_{N}+G^{*}-\Xi \leq 0,
$$

where $\chi=\sqrt{\lambda_{\max }\left(C^{\mathrm{T}} C\right)}, \quad \Xi=\operatorname{diag}\left(\xi_{1}, \xi_{2}, \cdots, \xi_{N}\right) \quad$ and $\quad G^{*}=\left(g_{i j}^{*}\right) \quad$ with $g_{i i}^{*}=c \varpi g_{i i}$ and $g_{i j}^{*}=\operatorname{cog}_{i j}(i \neq j)$. Then, under the controller (11), the controlled network (1) is said to be synchronized onto the target state (3) within a fixed time

$$
T=\frac{2 \phi}{\beta(\phi-\theta) 2^{\frac{\phi+\theta}{2 \phi}}}+\frac{2 l}{\gamma(l-k) 2^{\frac{l+k}{2 l}}(N n)^{\frac{l+k}{2 l}}} .
$$

Proof: Define a Lyapunov function as

$$
V=\frac{1}{2} \sum_{i=1}^{N} e_{i}^{\mathrm{T}}(t) e_{i}(t)
$$

Calculating the derivative of $V(t)$ along the trajectory of error system (4), we have

$$
\begin{aligned}
\dot{V}(t)= & \sum_{i=1}^{N} e_{i}^{\mathrm{T}}(t) A^{s} e_{i}(t)+\sum_{i=1}^{N} e_{i}^{\mathrm{T}}(t) F\left(\psi_{i}(t)\right) \\
& +c \sum_{i=1}^{N} \sum_{j=1}^{N} g_{i j} e_{i}^{\mathrm{T}}(t) \Gamma \psi_{j}(t)-\sum_{i=1}^{N} \epsilon_{i} e_{i}^{\mathrm{T}}(t) e_{i}(t) \\
& -\beta \sum_{i=1}^{N} e_{i}^{\mathrm{T}}(t) e_{i}^{\frac{\theta}{\phi}}(t)-\gamma \sum_{i=1}^{N} e_{i}^{\mathrm{T}}(t) e_{i}^{\frac{k}{l}}(t) .
\end{aligned}
$$

According to Lemmas 1 and Lemma 4, Equation (9) and Assumption 1, we have

$$
\begin{aligned}
& \dot{V}(t) \leq \lambda_{\max }\left(A^{s}\right) \sum_{i=1}^{N}\left\|e_{i}(t)\right\|^{2}+\beta \chi \sum_{i=1}^{N}\left\|e_{i}(t)\right\|^{2}+c \varpi \sum_{i=1}^{N} g_{i i}\left\|e_{i}(t)\right\|^{2} \\
&+c \varrho \sum_{i=1}^{N} \sum_{j=1, j \neq i}^{N} g_{i j}\left\|e_{i}(t)\right\| \cdot\left\|e_{j}(t)\right\|-\sum_{i=1}^{N} \epsilon_{i}\left\|e_{i}(t)\right\|^{2} \\
&-\beta \sum_{i=1}^{N} \sum_{j=1}^{N} e_{i j}^{\frac{\phi+\theta}{\phi}}(t)-\gamma \sum_{i=1}^{N} \sum_{j=1}^{N} e_{i j}{ }^{l+j}(t) \\
& \leq \lambda_{\max }\left(A^{s}\right) \sum_{i=1}^{N}\left\|e_{i}(t)\right\|^{2}+\beta \chi \sum_{i=1}^{N}\left\|e_{i}(t)\right\|^{2}+c \varpi \sum_{i=1}^{N} g_{i i}\left\|e_{i}(t)\right\|^{2} \\
&+c \rho \sum_{i=1}^{N} \sum_{j=1, j \neq i}^{N} g_{i j}\left\|e_{i}(t)\right\| \cdot\left\|e_{j}(t)\right\|-\sum_{i=1}^{N} \epsilon_{i}\left\|e_{i}(t)\right\|^{2}-\beta 2^{\frac{\phi+\theta}{2 \phi}} V^{\frac{\phi+\theta}{2 \phi}}(t) \\
&-\gamma 2^{\frac{l+k}{2 l}}(N n)^{\frac{l-k}{2 l}} V^{\frac{l+k}{2 l}}(t) .
\end{aligned}
$$


Denote $E=\left[\left\|e_{1}(t)\right\|,\left\|e_{2}(t)\right\|, \cdots,\left\|e_{N}(t)\right\|\right]^{\mathrm{T}}$, then

$$
\begin{aligned}
\dot{V}(t) \leq & E^{\mathrm{T}}\left\{\left(\lambda_{\max }\left(A^{s}\right)+\delta \chi\right) I_{n}+G^{*}-\Xi\right\} E \\
& -\beta 2^{\frac{\phi+\theta}{2 \phi}} V^{\frac{\phi+\theta}{2 \phi}}(t)-\gamma 2^{\frac{l+k}{2 l}}(N n)^{\frac{l-k}{2 l}} V^{\frac{l+k}{2 l}}(t) .
\end{aligned}
$$

By (12), it follows that

$$
\dot{V}(t) \leq-\beta 2^{\frac{\phi+\theta}{2 \phi}} V^{\frac{\phi+\theta}{2 \phi}}(t)-\gamma 2^{\frac{l+k}{2 l}}(N n)^{\frac{l-k}{2 l}} V^{\frac{l+k}{2 l}}(t) .
$$

By employing Lemma 4, the following estimated value of settling time can be achieved

$$
T=\frac{2 \phi}{\beta(\phi-\theta) 2^{\frac{\phi+\theta}{2 \phi}}}+\frac{2 l}{\gamma(l-k) 2^{\frac{l+k}{2 l}}(N n)^{\frac{l+k}{2 l}}} .
$$

This completes the proof.

Corollary 3: Suppose Assumption 1 holds and output matrix $C=I_{N}$. If there exists positive constants $\xi_{i}, i=1,2, \cdots, N$, such that

$$
\left(\lambda_{\max }\left(A^{s}\right)+\delta\right) I_{N}+G^{*}-\Xi \leq 0,
$$

where $\Xi=\operatorname{diag}\left(\xi_{1}, \xi_{2}, \cdots, \xi_{N}\right) \quad$ and $\quad G^{*}=\left(g_{i j}^{*}\right) \quad$ with $\quad g_{i i}^{*}=c \varpi g_{i i} \quad$ and $g_{i j}^{*}=\operatorname{c\varrho g}_{i j}(i \neq j)$. Then complex network (1) will realize fixed-time synchronization under the controller (11).

Remark 8: It can be found that from the results of Theorem 2 that the settling time is independent on the initial value of state $x_{i}(0)$ and $x_{0}(0)$. Furthermore, the settling time can be estimated by the dimension of node $n$, the design parameters and the group order $\mathrm{N}$.

Remark 9: In reality, one can comprehend the theoretical basis why we design controller 11 from Theorem 1 and the proof process of Theorem 1. When $\beta=\gamma=0$, the controller $u_{i}(t)=-\xi_{i} e_{i}(t)$ which is widely used in [25] can ensure that the complex networks 1 achieves asymptotical synchronization that can be seen from (12) where $\dot{V}(t) \leq E^{\mathrm{T}}\left\{\left(\lambda_{\max }\left(A^{s}\right)+\delta\right) I_{n}+\hat{G}^{*}-\Xi\right\} E$, where ma$\operatorname{trix}\left(\lambda_{\max }\left(A^{s}\right)+\delta\right) I_{n}+\hat{G}^{*}-\Xi$ is negative definite. As for they part $-\beta e_{i}^{p}$ and the part $-\gamma e_{i}^{q}$, they play the role which control the complex networks such that $V(t)=1$ in a fixed time $T_{1}$ when $V(t)>1$ and $V(t)$ approaches to zero in a fixed time $T_{2}$ when $V(t) \leq 1$ respectively. Therefore, to some degree, the settle time $\mathrm{T}$ is the sum of $T_{1}$ and $T_{2}$.

Remark 10: It is clear that the settling time of Theorem 2 is different from the settling time of Theorem 1 and results in [5] [9] which are dependent on the initial value of $e_{i}(0)$. What's more, we can easily find that the part $\beta e_{i}^{\frac{\theta}{\phi}}(t)$ and the part $-\gamma e_{i}^{\frac{k}{l}}(t)$ play different roles to realize the fixed-time synchronization based on the analysis. 
Consider the following for comparison purpose:

$$
\left\{\begin{array}{l}
\dot{h}(t)=\left\{\begin{array}{lc}
-2 \bar{\beta} h^{\frac{\phi+\theta}{2 \phi}}(t), & h(t) \geq 1, \\
-2 \bar{\gamma} h^{\frac{l+k}{2 l}}(t), & 0<h(t)<1, \\
0, & h(t)=0,
\end{array}\right. \\
h(0)=V(0) .
\end{array}\right.
$$

where $\bar{\beta}=\beta 2^{\frac{\theta-\phi}{2 \phi}}, \bar{\gamma}=\gamma 2^{\frac{k-l}{2 l}}(N n)^{\frac{l-k}{2 l}}$. Compared (13) with (14), it can be easily seen that $0 \leq V(t) \leq h(t)$. Consequently, to prove the fixed-time stability of (14), we just only need to study the corresponding problem of the zero solution of the above system (14).

Let $z(t)=h^{\frac{\phi-\theta}{2 \phi}}(t)$ when $h(t) \geq 1$. Then

$$
\left\{\begin{array}{l}
\dot{z}(t)=\bar{\beta}\left(\frac{\theta}{\phi}-1\right), 0<z(t) \leq 1 \\
z(0)=z_{0}=h_{0}^{\frac{\phi-\theta}{2 \phi}} .
\end{array}\right.
$$

From (14) and (15), it can be seen that $z(0) \rightarrow 0$ when $h(0) \rightarrow \infty$, and $z(t) \rightarrow 1$ for $z(t) \rightarrow 1$.

Let $z(t)=h^{\frac{l-k}{2 l}}(t)$ when $0 \leq h(t)<1$. Then

$$
\left\{\begin{array}{l}
\dot{z}(t)=-\bar{\gamma}\left(1-\frac{k}{l}\right), 0 \leq z(t)<1 \\
z(0)=1 .
\end{array}\right.
$$

Obviously, it follows that $z(t) \rightarrow 1$ when $h(t) \rightarrow 1$, and $z(t) \rightarrow 0$ for $z(t) \rightarrow 0$.

Accordingly, the global stability of the zero solution of (14) within fixed time has been transformed to the following two problems: 1) the solution of (15) approaches to 1 in a fixed time $\left.T_{a} ; 2\right)$ the solution of (16) reaches 0 from 1 in a fixed time $T_{b}$. Then, $z(t) \rightarrow 0$ in a fixed time $T=T_{a}+T_{b}$ from any initial value $z(0)$.

Remark 11: Actually, the fixed time $T$ can be obtained easily if the fixed time $T_{\mathrm{a}}$ and $T_{\mathrm{b}}$ can be achieved. In [29], a new approach to obtain the settling time was presented according to above analysis.

\section{Adaptive Adjustment of the Coupling Strength}

To our best knowledge, the adaptive coupling strength problem for fixed-time synchronization of complex networks with output nodes is less investigated. Therefore, with the help of adaptive technique, the coupling strength adaptive adjustment will be discussed. Associated with the adaptive coupling law, the controlled complex network with output nodes is leaded to: 


$$
\left\{\begin{array}{l}
\dot{x}_{i}(t)=A x_{i}(t)+f\left(y_{i}(t)\right)+c(t) \sum_{j=1}^{N} g_{i j} \Gamma y_{i}(t)+u_{i}(t), \\
y_{i}(t)=C x_{i}(t), \quad i \in \mathbb{N}, \\
\dot{c}(t)=\alpha \sum_{i=1}^{N} e_{i}^{\mathrm{T}} \Gamma C e_{i}(t) .
\end{array}\right.
$$

where $\alpha$ is a small positive constant and $u_{i}(t)$ are same as (11).

Theorem 3: Suppose that Assumption 1 holds and $Г C$ is a positive define matrix. Then, the adaptively controlled complex networks can achieve fixed-time synchronization with a desirable coupling strength.

Proof: Define a Lyapunov function as

$$
V=\frac{1}{2} \sum_{i=1}^{N} e_{i}^{\mathrm{T}}(t) e_{i}(t)+\frac{1}{2 \alpha}(c(t)-\tilde{c})^{2}
$$

Calculating the derivative of $V(t)$ along the trajectory of error system (8), it follows that

$$
\begin{gathered}
\dot{V}(t)=\sum_{i=1}^{N} e_{i}^{\mathrm{T}}(t) \dot{e}_{i}(t)+\frac{1}{\alpha}(c(t)-\tilde{c}) \dot{c}(t) \\
=\sum_{i=1}^{N} e_{i}^{\mathrm{T}}(t)\left\{A e_{i}(t)+f\left(y_{i}(t)\right)-f\left(y_{0}(t)\right)\right. \\
\left.+c \sum_{j=1}^{N} g_{i j} \Gamma \psi_{j}(t)-\xi_{i} e_{i}(t)-\beta e_{i}^{\frac{\theta}{\phi}}(t)-\gamma e_{i}^{\frac{k}{l}}(t)\right\} \\
+(c(t)-\tilde{c}) \sum_{i=1}^{N} e_{i}^{\mathrm{T}}(t) \Gamma C e_{i}(t) \\
\leq \sum_{i=1}^{N} e_{i}^{\mathrm{T}}(t) A e_{i}(t)+\sum_{i=1}^{N} e_{i}^{T}(t)\left(f\left(y_{i}(t)\right)-f\left(y_{0}(t)\right)\right) \\
-\sum_{i=1}^{N} \epsilon_{i} e_{i}^{\mathrm{T}}(t) e_{i}(t)-\tilde{c} \sum_{i=1}^{N} e_{i}^{\mathrm{T}}(t) \Gamma C e_{i}(t) \\
+c(t) \sum_{i=1}^{N} \sum_{j=1}^{n} e_{i}^{\mathrm{T}}(t) g_{i j} \Gamma C e_{i}(t)+c(t) \sum_{i=1}^{N} e_{i}^{\mathrm{T}}(t) \Gamma C e_{i}(t) \\
-\beta \sum_{i=1}^{N} e_{i}^{\mathrm{T}}(t) e_{i}^{\frac{\theta}{\phi}}(t)-\gamma \sum_{i=1}^{N} e_{i}^{\mathrm{T}}(t) e_{i}^{\frac{k}{l}}(t) \\
\leq \lambda_{\max }\left(A^{s}\right) \sum_{i=1}^{N} e_{i}^{\mathrm{T}}(t) e_{i}(t)+\chi \sum_{i=1}^{N} e_{i}^{\mathrm{T}}(t) e_{i}(t) \\
-\sum_{i=1}^{N} \epsilon_{i} e_{i}^{\mathrm{T}}(t) e_{i}(t)+e^{\mathrm{T}}\left(\Gamma C \otimes\left(c(t)\left(G^{s}+I_{N}\right)-\tilde{c} I_{N}\right)\right) e \\
-\beta 2^{\frac{\phi+\theta}{2 \phi}} V^{\frac{\phi+\theta}{2 \phi}}(t)-\gamma 2^{\frac{l+k}{2 l}}(N n)^{\frac{l-k}{2 l}} V^{\frac{l+k}{2 l}}(t) .
\end{gathered}
$$

where $e^{\mathrm{T}}=\left(e_{1}(t), e_{2}(t), \cdots, e_{n}(t)\right)$. Then

$$
\begin{aligned}
\dot{V}(t) \leq & e^{T}\left\{\left(\lambda_{\max }\left(A^{s}\right)+\beta \chi\right) I_{n}-\Xi\right\} e \\
& +e^{T}\left(\Gamma C \otimes\left(c(t)\left(G^{s}+I_{N}\right)-\tilde{c} I_{N}\right)\right) e \\
& -\beta 2^{\frac{\phi+\theta}{2 \phi}} V^{\frac{\phi+\theta}{2 \phi}}(t)-\gamma 2^{\frac{l+k}{2 l}}(N n)^{\frac{l-k}{2 l}} V^{\frac{l+k}{2 l}}(t) .
\end{aligned}
$$


Obviously, we can pick suitable $\epsilon_{i}, i=1,2, \cdots, N$, such that

$$
\left(\lambda_{\max }\left(A^{s}\right)+\delta \chi\right) I_{n}-\Xi<0
$$

Meanwhile, we also can pick suitable $\tilde{C}$ such that

$$
c(t)\left(G^{s}+I_{N}\right)-\tilde{c} I_{N}<0 .
$$

According to the property of $K$ product [5], namely, if $c(t)\left(G+I_{N}\right)-\tilde{c} I_{N}<0$ and $\Gamma C>0$, then one can easily obtain that

$$
\Gamma C \otimes\left(c(t)\left(G^{s}+I_{N}\right)-\tilde{c} I_{N}\right)<0 .
$$

Then, the following inequality holds:

$$
\dot{V}(t) \leq-\beta 2^{\frac{1+p}{2}} V^{\frac{1+p}{2}}(t)-\gamma 2^{\frac{1-q}{2}}(N n)^{\frac{1-q}{2}} V^{\frac{1-q}{2}}(t) .
$$

It indicates that $e_{i}(t) \rightarrow 0, i=1,2, \cdots, N$ within fixed time and consequently the adaptively controlled complex networks can achieve fixed-time synchronization. Besides, $\dot{c}(t) \rightarrow 0, i=1,2, \cdots, N$. it means that $c(t) \rightarrow c_{0}$, here, the $c_{0}$ is a nonnegative constant. The proof is completed.

\section{Numerical Examples}

In this section, two numerical examples are presented to show the effectiveness of the proposed theoretical analysis. Specifically, Example 1 is given to verify Theorem 1 and Example 2 is provided to illustrate Theorem 2.

Now, we consider the following two-dimensional van der pol system [21] which is presented as:

$$
\left\{\begin{array}{l}
\dot{x}_{i}(t)=A x_{i}(t)+f\left(y_{i}(t)\right) \\
y_{i}(t)=C x_{i}(t), \quad i \in \mathbb{N},
\end{array}\right.
$$

where $x_{i}^{\mathrm{T}}(t)=\left(x_{i 1}(t), x_{i 2}(t)\right)$ is the state vector and

$$
\begin{gathered}
A=\left(\begin{array}{ll}
0 & 0 \\
0 & 1
\end{array}\right) \\
f^{\mathrm{T}}\left(y_{i}(t)\right)=\left(f_{1}\left(y_{i}(t)\right), f_{2}\left(y_{i}(t)\right)\right)=\left(\ell\left(y_{i}(t)-\frac{1}{3}\left(y_{i}(t)\right)\right)^{3},-y_{i}(t) / \ell\right),(19)
\end{gathered}
$$

and $C=[1,1]$. The system (18) is a van der Pol oscillator when $\ell=1$, and its trajectories are bounded.

\subsection{Example 1}

Consider CNs with output nodes as

$$
\left\{\begin{array}{l}
\dot{x}_{i}(t)=A x_{i}(t)+f\left(y_{i}(t)\right)+c \sum_{j=1}^{10} g_{i j} \Gamma y_{i}(t)+u_{i}(t) \\
y_{i}(t)=C x_{i}(t), \quad i=1, \cdots, 10,
\end{array}\right.
$$

where 


$$
G=\left[\begin{array}{cccccccccc}
-1 & 1 & 0 & 0 & 0 & 0 & 0 & 0 & 0 & 0 \\
1 & -2 & 0 & 1 & 0 & 0 & 0 & 0 & 0 & 0 \\
0 & 1 & -1 & 0 & 0 & 0 & 0 & 0 & 0 & 0 \\
1 & 0 & 0 & -2 & 1 & 0 & 0 & 0 & 0 & 0 \\
0 & 0 & 1 & 0 & -1 & 0 & 0 & 0 & 0 & 0 \\
0 & 0 & 0 & 1 & 0 & -3 & 0 & 1 & 0 & 1 \\
0 & 1 & 0 & 1 & 0 & 0 & -2 & 0 & 0 & 0 \\
1 & 0 & 0 & 0 & 0 & 1 & 1 & -3 & 0 & 0 \\
1 & 0 & 0 & 0 & 0 & 1 & 1 & 0 & -3 & 0 \\
0 & 0 & 1 & 0 & 1 & 0 & 0 & 1 & 0 & -3
\end{array}\right] .
$$

Selecting the inner-coupling matrix $\Gamma=[1,1]^{\mathrm{T}}$ and the coupling strength $c=1$, we design the synchronization controller as

$$
u_{i}(t)=-\epsilon_{i} e_{i}(t)-\beta e_{i}^{\frac{\theta}{\phi}}(t), i=1, \cdots, 10,
$$

We will control the CNs (18) under controllers (21) such that it will be synchronized onto the following target state within finite time:

$$
\left\{\begin{array}{l}
\dot{x}_{0}(t)=A x_{0}(t)+f\left(y_{0}(t)\right), \\
y_{0}(t)=C x_{0}(t) .
\end{array}\right.
$$

From the analysis of [21], for the function $f(\cdot)$, the parameter $\delta$ can be selected as 21.22 which can satisfy Assumption 1.

Take $x_{1}(0)=(-6.321,2)^{\mathrm{T}}, \quad x_{2}(0)=(-4.321,4)^{\mathrm{T}}, \quad x_{3}(0)=(-2.321,6)^{\mathrm{T}}$, $x_{4}(0)=(-0.321,8)^{\mathrm{T}}, \quad x_{5}(0)=(1.679,10)^{\mathrm{T}}, \quad x_{6}(0)=(3.679,12)^{\mathrm{T}}$, $x_{7}(0)=(5.679,14)^{\mathrm{T}}, \quad x_{8}(0)=(7.679,16)^{\mathrm{T}}, \quad x_{9}(0)=(9.679,18)^{\mathrm{T}}$, $x_{10}(0)=(11.679,20)^{\mathrm{T}}$ and $x_{0}(0)=(0.065,0.1)^{\mathrm{T}}$.

Take $\theta=1, \phi=3$ and step-length $=0.0001$. Actually, as long as $\xi=\min \left\{\xi_{1}, \xi_{2}, \cdots, \xi_{10}\right\}$ sufficiently large, the CNs (18) are finite-timely synchronized onto (22) under controller (21). In the following numerical simulations, we take

$$
\Xi=\operatorname{diag}(55.369,59.341,60.33,55.57,66.01,70.03,71.44,77.2,87.21,88.36) \text {. }
$$

Take $\theta=1, \phi=3, \beta=5$, we get $T_{0}=0.071$. Figure 1 , Figure 2 show the time response of error systems without control and Figure 3 and Figure 4 present that the synchronization is achieved in finite time.

\subsection{Example 2}

Consider the networks (21), similar to numerical simulation of Example 1, fixedtime synchronization controllers are designed as follows:

$$
u_{i}(t)=-\epsilon_{i} e_{i}-\beta e_{i}^{\frac{\phi}{\theta}}-\gamma e_{i}^{\frac{k}{l}}(t), i=1, \cdots, 10 .
$$

Take $x_{1}(0)=(-6.321,2)^{\mathrm{T}}, \quad x_{2}(0)=(-4.321,4)^{\mathrm{T}}, \quad x_{3}(0)=(-2.321,6)^{\mathrm{T}}$, $x_{4}(0)=(-0.321,8)^{\mathrm{T}}, \quad x_{5}(0)=(1.679,10)^{\mathrm{T}}, \quad x_{6}(0)=(3.679,12)^{\mathrm{T}}$, $x_{7}(0)=(5.679,14)^{\mathrm{T}}, \quad x_{8}(0)=(7.679,16)^{\mathrm{T}}, \quad x_{9}(0)=(9.679,18)^{\mathrm{T}}$, $x_{10}(0)=(11.679,20)^{\mathrm{T}}$ and $x_{0}(0)=(0.065,0.1)^{\mathrm{T}}$. 
Take $k=5, l=3$ and step-length $=0.00005$, other relative parameters are as same as Example 1. Take $\beta=\gamma=5$, we obtain settling time $T=0.06861$. Figure 5 and Figure 6 show the time response of error systems without controller and Figure 7 and Figure 8 present that the fixed-time synchronization is realized.

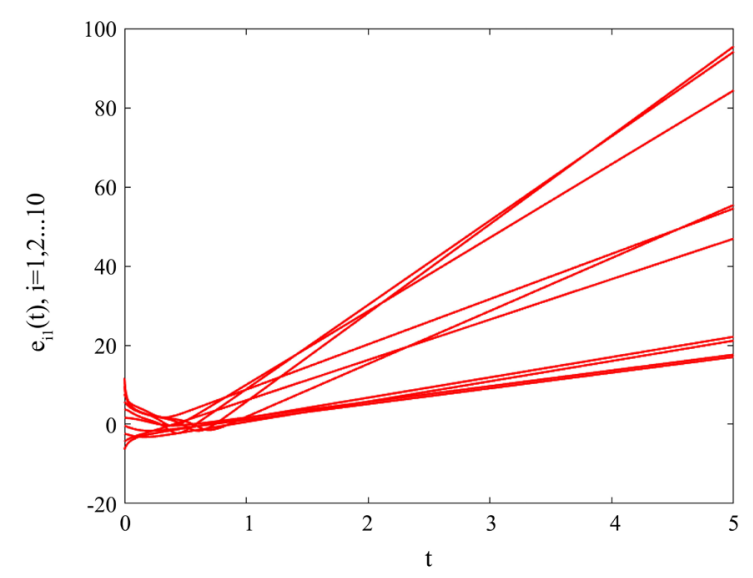

Figure 1. Time response of synchronization errors without controller (21).

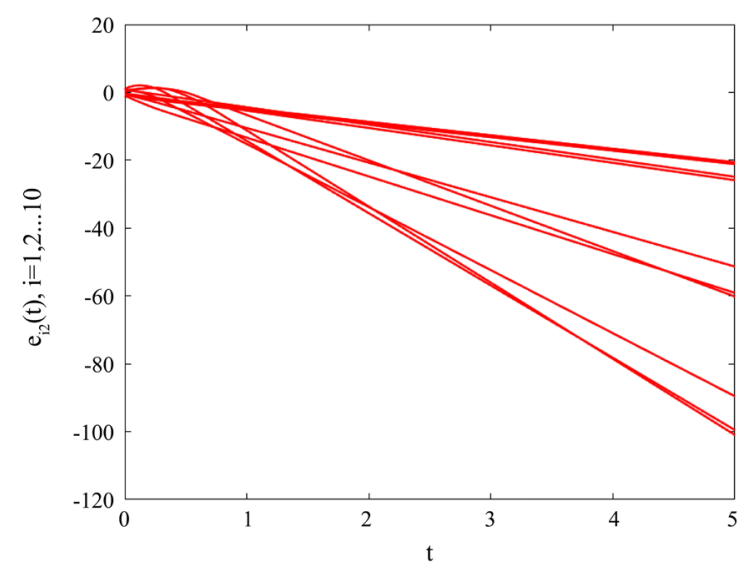

Figure 2. Time response of synchronization errors without controller (21).

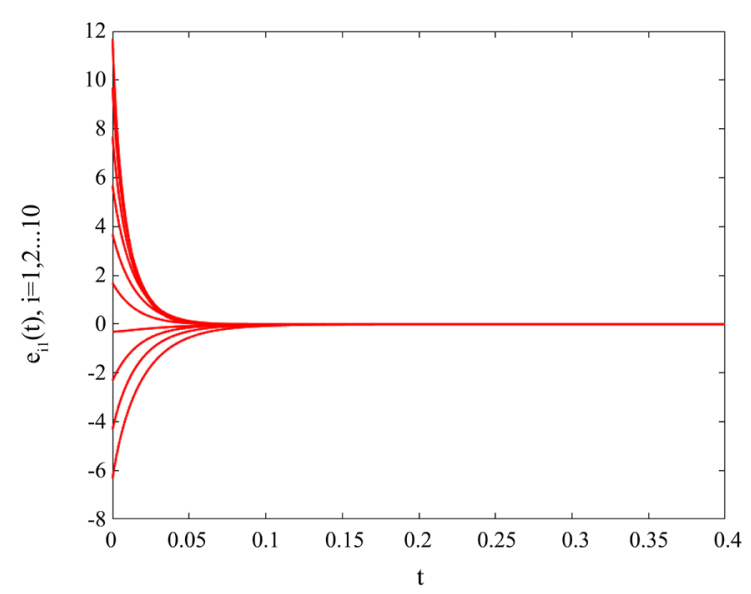

Figure 3. Time response of synchronization errors under controllers (21) with $\theta=1, \phi=3, \beta=5$. 


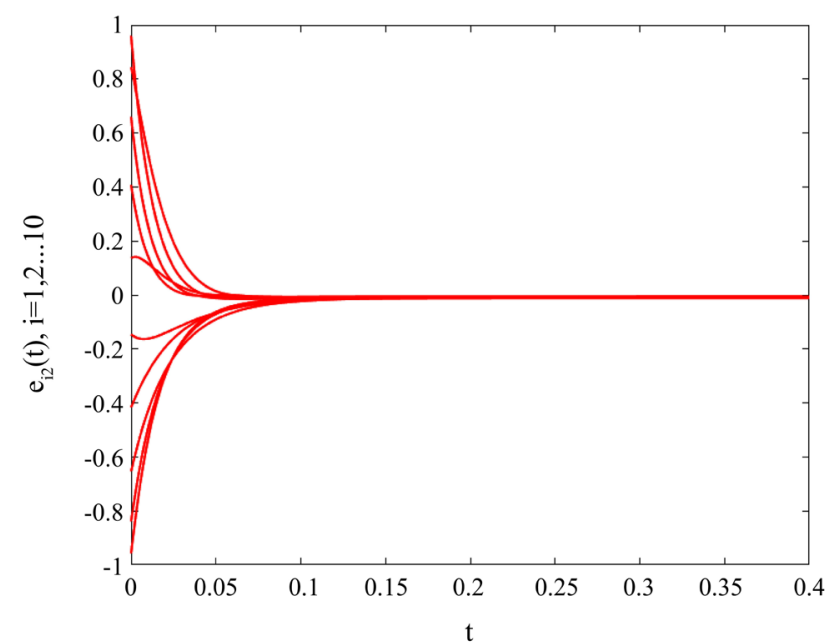

Figure 4. Time response of synchronization errors under controllers (21) with $\theta=1, \phi=3, \beta=5$.

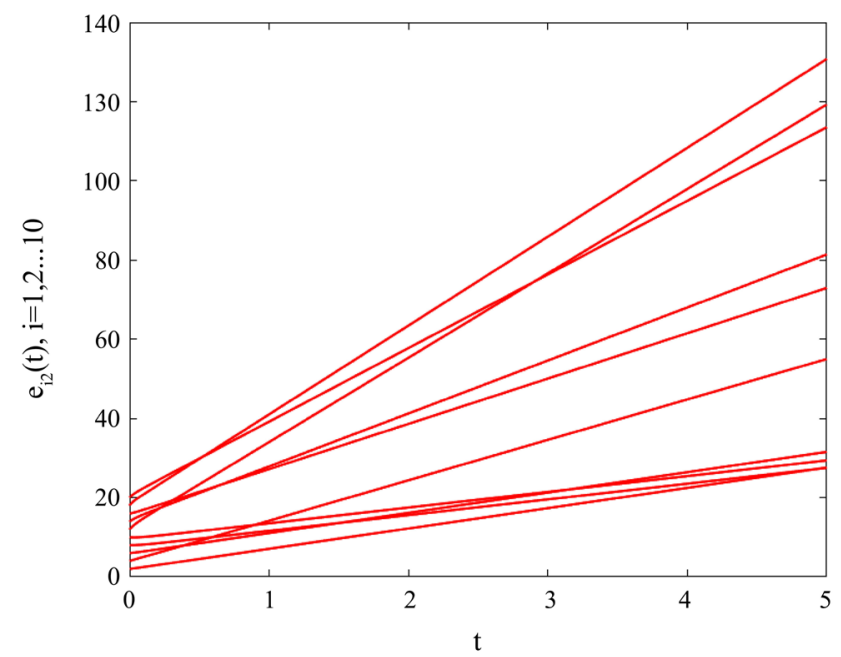

Figure 5. Time response of synchronization errors without controller (23).

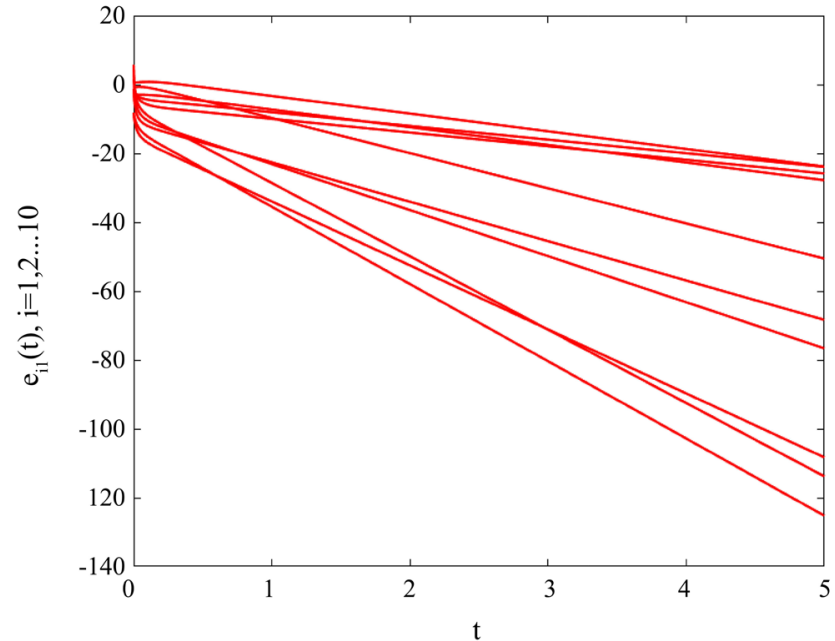

Figure 6. Time response of synchronization errors without controller (23). 


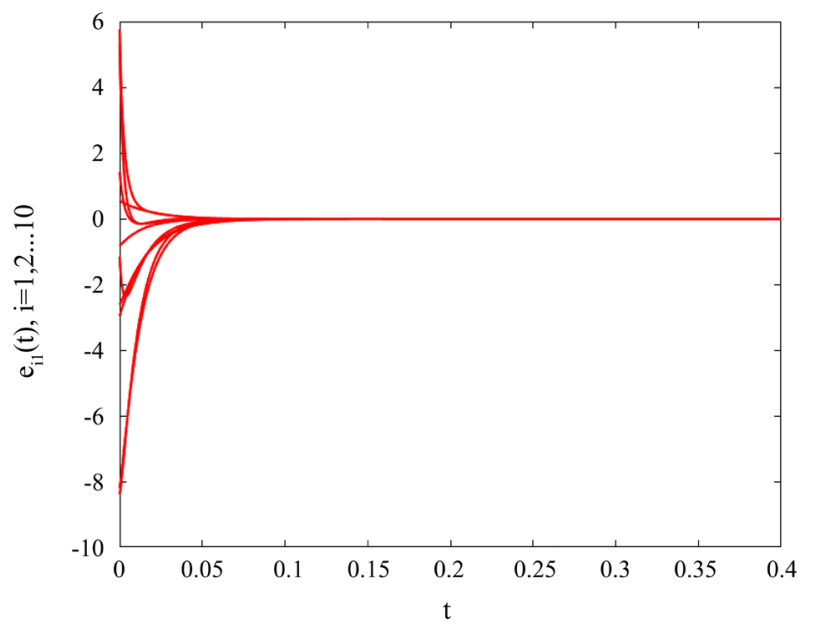

Figure 7. Time response of synchronization errors under controllers (23) with $\theta=1, \phi=3, k=5, l=3, \beta=5, \gamma=5$.

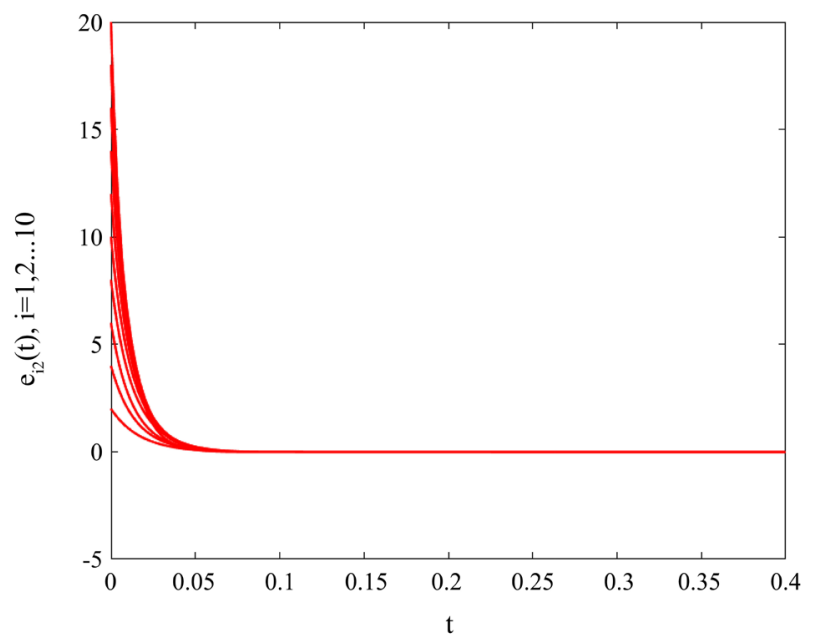

Figure 8. Time response of synchronization errors under controllers (23) with $\theta=1, \phi=3, k=5, l=3, \beta=5, \gamma=5$.

\section{Conclusion}

In this paper, the issue of the fixed-time and the finite-time synchronization for a class of general output-coupling complex networks with output feedback nodes are discussed. Under the novel continuous controllers, the chattering phenomenon is eliminated and the fixed-time and finite-time synchronization are achieved as well. Then, the adaptive coupling strength problem for fixed-time synchronization of complex networks with output nodes is investigated. Finally, the availability of proposed fixed-time and finite-time synchronization frame are demonstrated by several numerical examples.

\section{Conflicts of Interest}

The author declares no conflicts of interest regarding the publication of this paper. 


\section{References}

[1] Stogatz, S. and Stewart, I. (1993) Coupled Oscillators and Biological Synchronization. Scientific American, 269, 102-109. https://doi.org/10.1038/scientificamerican1293-102

[2] Strogatz, S.H. (2001) Exploring Complex Networks. Nature, 410, 268-276. https://doi.org/10.1038/35065725

[3] Xie, Q., Chen, R.G. and Bolt, E. (2002) Hybrid Chaos Synchronization and Its Application in Information Processing. Mathematical and Computer Modelling, 1, 145-163. https://doi.org/10.1016/S0895-7177(01)00157-1

[4] Gray, C.M. (1994) Synchronous Oscillations in Neural Systems. Journal of Computational Neuroscience, 1, 11-38. https://doi.org/10.1007/BF00962716

[5] Yu, W.W., Chen, G.R. and Lv, J.H. (2009) On Pinning Synchronization of Complex Dynamical Networks. Automatica, 45, 429-435. https://doi.org/10.1016/j.automatica.2008.07.016

[6] Zhang, W., Li, C., Huang, T. and He, X. (2015) Synchronization of Memristor-Based Coupling Recurrent Neural Networks with Time-Varying Delays and Impulses. IEEE Transactions on Neural Networks and Learning Systems, 26, 3308-3313. https://doi.org/10.1109/TNNLS.2015.2435794

[7] Han, F., Wei, G.L., Ding, D.R. and Song, Y. (2017) Finite-Horizon Bounded H Synchronisation and State Estimation for Discrete-Time Complex Networks: Local Performance Analysis. IET Control Theory and Applications, 11, 827-837. https://doi.org/10.1049/iet-cta.2016.1161

[8] Shen, B., Wang, Z.D., Ding, D.R. and Shu, H.S. (2013) H-Infinity State Estimation for Complex Networks with Uncertain Inner Coupling and Incomplete Measurements. IEEE Transactions on Neural Networks and Learning Systems, 24, 2027-2037. https://doi.org/10.1109/TNNLS.2013.2271357

[9] Wu, W., Zhou, W.J. and Chen, T.P. (2009) Cluster Synchronization of Linearly Coupled Complex Networks under Pinning Control. IEEE Transactions on Circuits and Systems I: Regular Papers, 56, 829-839. https://doi.org/10.1109/TCSI.2008.2003373

[10] Lam, H. and Leung, F. (2006) Synchronization of Uncertain Chaotic Systems Based on the Fuzzy-Model-Based Approach. International Journal of Bifurcation and Chaos, 16, 1435-1444. https://doi.org/10.1142/S0218127406015404

[11] Guan, Z., Liu, Z., Feng, G. and Wang, Y. (2010) Synchronization of Complex Dynamical Networks with Time-Varying Delays via Impulsive Distributed Control. IEEE Transactions on Circuits and Systems I: Regular Papers, 57, 2182-2195. https://doi.org/10.1109/TCSI.2009.2037848

[12] Zhang, L., Yang, X.S., Chen, X. and Feng, J.W. (2017) Exponential Synchronization of Complex-Valued Complex Networks with Time-Varying Delays and Stochastic Perturbations Via Time-Delayed Impulsive Control. Applied Mathematics and Computation, 306, 22-30. https://doi.org/10.1016/j.amc.2017.02.004

[13] Bao, H., Park, J. and Cao, J. (2016) Exponential Synchronization of Coupled Stochastic Memristor-Based Neural Networks with Time-Varying Probabilistic Delay Coupling and Impulsive Delay. IEEE Transactions on Neural Networks and Learning Systems, 27, 190-201. https://doi.org/10.1109/TNNLS.2015.2475737

[14] Yang, X., Wu, Z. and Cao, J.D. (2013) Finite-Time Synchronization of Complex Networks with Nonidentical Discontinuous Nodes. Nonlinear Dynamics, 73, 2313 2327. https://doi.org/10.1007/s11071-013-0942-4 
[15] Jiang, S.Q., Lu, X.B., Xie, C. and Cai, S.M. (2017) Adaptive Finite-Time Control for Overlapping Ciuster Synchronization in Coupled Complex Networks. Neurocomputing, 266, 188-195. https://doi.org/10.1016/j.neucom.2017.05.031

[16] Mei, J., Jiang, M., Wu, Z. and Wang, X. (2015) Periodically Intermittent Controlling for Finite-Time Synchronization of Complex Dynamical Networks. Nonlinear Dynamics, 79, 295-305. https://doi.org/10.1007/s11071-014-1664-y

[17] Liu, M., Jiang, H.J. and Hu, C. (2017) Finite-Time Synchronization of Delayed Dynamical Networks via Aperiodically Intermittent Control. Journal of the Franklin Institute, 4, 5374-5397. https://doi.org/10.1016/j.jfranklin.2017.05.030

[18] Liu, X.W. and Chen, T.P. (2018) Finite-Time and Fixed-Time Cluster Synchroniozation with or without Pinning Control. IEEE Transactions on Cybernetics, 48, 950-955. https://doi.org/10.1109/TCYB.2016.2630703

[19] Zhang, W.L., Li, C.D., Huang, T.W. and Huang, J.J. (2018) Fixed-Time Synchronization of Complex Networks with Nonidentical Nodes and Stochastic Noise Perturbations. Physica A, 492, 1531-1542. https://doi.org/10.1016/j.physa.2017.11.079

[20] Liu, J., Yu, Y., Wang, Q. and Sun, C.Y. (2017) Fixed-Time Event-Triggered Consensus Control for Multi-Agent Systems with Nonlinear Uncertainties. Neurocomputing, 260, 497-504. https://doi.org/10.1016/j.neucom.2017.04.061

[21] Li, Q.B., Guo, J., Sun, C.Y. and Wu Y.Y. (2017) Adaptive Synchronization for a Class of Output-Coupling Complex Networks with Output Feedback Nodes. IET Control Theory, 11, 3372-3380. https://doi.org/10.1049/iet-cta.2017.0568

[22] Hong, Y., Wang, J. and Cheng, D. (2006) Adaptive Finite-Time Control of Nonlinear Systems with Parametric Uncertainty. IEEE Transactions on Automatic Control, 51, 858-862. https://doi.org/10.1109/TAC.2006.875006

[23] Aghababa, M. and Agahababa, H. (2012) Adaptive Finite-Time Synchronization of Non-Autonomous Chaotic Systems with Uncertainty. Journal of Computational and Nonlinear Dynamics, 8, Article ID: 031006. https://doi.org/10.1115/1.4023007

[24] Yang, X. and Hu, J. (2016) Finite-Time Synchronization of Coupled Networks with Markovian Topology and Impulsive Effects. IEEE Trans Automatic Control, 61, 22562261. https://doi.org/10.1109/TAC.2015.2484328

[25] Lu, W.L. and Chen, T.P. (2004) Synchronization of Coupled Connected Neural Networks with Delays. IEEE Transactions on Circuits and Systems I: Regular Papers, 51, 2494-2503. https://doi.org/10.1109/TCSI.2004.838308

[26] Chen, T.P., Liu, X.W. and Lu, W.L. (2007) Pinning Complex Networks by a Single Controller. IEEE Transactions on Circuits and Systems I: Regular Papers, 54, 13171326. https://doi.org/10.1109/TCSI.2007.895383

[27] Liu, X. and Chen, T. (2015) Fixed-Time Cluster Synchronization for Complex Networks via Pinning Control. arXiv:1509.03350.

[28] Yang, X. and Cao, J. (2010) Finite-Time Stochastic Synchronization of Complex Networks. Applied Mathematical Modelling, 34, 3631-3641. https://doi.org/10.1016/j.apm.2010.03.012

[29] Yang, X., Lam, J., Ho, D. and Feng, Z. (2017) Fixed-Time Cluster Synchronization for Complex Networks with Impulsive Effects via Non-Chattering Control. IEEE Trans Automatic Control, 62, 5511-5521.

https://doi.org/10.1109/TAC.2017.2691303 